\title{
Monkey Supplementary Eye Field Neurons Signal the Ordinal Position of Both Actions and Objects
}

\author{
Tamara K. Berdyyeva ${ }^{1,2}$ and Carl R. Olson ${ }^{1,2}$ \\ ${ }^{1}$ Center for the Neural Basis of Cognition, Mellon Institute, and 2Department of Biological Sciences, Carnegie Mellon University, Pittsburgh, Pennsylvania
} 15213

When a monkey executes a learned series of eye movements (for example, rightward followed by upward followed by leftward), neurons in the supplementary eye field (SEF) fire differentially in conjunction with the first, second, and third movements. It has not been clear whether such ordinal position signals are truly general, accompanying all forms of sequential behavior, or accompany only learned sequences of movements. To resolve this issue, we trained monkeys to perform both a serial action task (making saccades in a fixed sequence of directions) and a serial object task (making saccades to a fixed sequence of objects). We found concordant ordinal position selectivity in the two tasks. Neuronal selectivity for the passage of time and expectation of reward could not explain such concordance. We conclude that SEF neurons signal ordinal position consistently across different task contexts. These signals presumably underlie the ability of primates including humans to perform a broad range of serial order tasks.

Key words: monkey; supplementary eye field; neuronal activity; serial order; reward; context independent

\section{Introduction}

The ability of humans to appreciate serial order underlies a large variety of cognitive functions, including planning, logic, and language production (Lashley, 1951; Dominey et al., 2003; Hoen et al., 2003). It has often been suggested that our ability to perform serial order tasks is mediated by neurons that systematically change their firing rate according to position in the sequence (for review, see Tanji, 2001; Rhodes et al., 2004; Ashe et al., 2006). The existence of such rank-selective neurons has been demonstrated in numerous regions of the brain that control motor behavior [caudate nucleus: Kermadi and Joseph (1995); globus pallidus: Mushiake and Strick (1995); primary motor cortex: Carpenter et al. (1999), Crowe et al. (2004), Lu and Ashe (2005); premotor cortex: Ohbayashi et al. (2003); anterior cingulate cortex: Akkal et al. (2002), Procyk and Joseph (2001); prefrontal cortex: Averbeck et al. (2002, 2003), Funahashi et al. (1997), Inoue and Mikami (2006); pre-supplementary motor area (pre-SMA) and supplementary motor area (SMA): Akkal et al. (2002), Clower and Alexander (1998), Lee and Quessy (2003), Shima and Tanji (2000), Sohn and Lee (2007); and supplementary eye field (SEF): Isoda and Tanji (2002, 2003), Lu et al. (2002)]. Previous studies of rank selectivity focused specifically on the ordinal position of a movement in a sequence of movements; however, such motor sequences are only a subset of a broader range of serial order behaviors. Neurons representing serial order at a fully abstract level should signal ordinal position not only in a learned sequence of

Received 0ct. 6, 2008; revised Nov. 12, 2008; accepted Dec. 3, 2008.

This work was supported by National Institutes of Health Grants R01 EY018620, P50 MH45156, P30 EY08098, and P41RR03631. We thank Karen McCracken for excellent technical assistance.

Correspondence should be addressed to Carl R. Olson, Department of Biological Sciences, Carnegie Mellon University, Pittsburgh, PA 15213. E-mail: tberdyye@andrew.cmu.edu.

DOI:10.1523/JNEUROSCI.4803-08.2009

Copyright $\odot 2009$ Society for Neuroscience $\quad$ 0270-6474/09/290591-09\$15.00/0 movements but also in other sequences, for example, during selection of objects in a learned sequence. No previous study has addressed the question whether rank-selective neurons are specialists, representing serial position in the context only of a movement sequence, or generalists, representing serial position in other contexts as well. To address this issue, we monitored the activity of SEF neurons during performance of both a serial action task and a serial object task. We found that rank-selective SEF neurons are generalists, representing serial position in both task contexts. To establish beyond doubt that these neurons represent serial order position, we performed novel control experiments assessing their sensitivity to factors that commonly are correlated with rank (the approach of reward and the passage of time). We found that they were genuinely sensitive to serial order position as distinct from these extraneous factors.

\section{Materials and Methods \\ General methods}

Subjects

Two adult male rhesus monkeys were used (Macaca mulatta; laboratory designations $\mathrm{O}$ and $\mathrm{T}$; monkey $\mathrm{O}$ was previously extensively trained on the variety of manual and oculomotor tasks; monkey T was naive). Experimental procedures were approved by the Carnegie Mellon University Animal Care and Use Committee and were in compliance with the guidelines set forth in the United States Public Health Service Guide for the Care and Use of Laboratory Animals.

Preparatory surgery

At the outset of the training period, each monkey underwent sterile surgery under general anesthesia maintained with isoflurane inhalation. The top of the skull was exposed, bone screws were inserted around the perimeter of the exposed area, a continuous cap of rapidly hardening acrylic was laid down so as to cover the skull and embed the heads of the screws, a head-restraint bar was embedded in the cap and scleral search coils were implanted on the eyes, with the leads directed subcutaneously 
to plugs on the acrylic cap. After initial training, a $2 \mathrm{~cm}$ diameter disk of acrylic and skull, centered on the midline of the brain approximately at anterior $21 \mathrm{~mm}$ (Horsely-Clarke reference frame) was removed. A cylindrical recording chamber was cemented into the hole with its base flush to the exposed dural membrane.

\section{Single-unit recording}

At the beginning of each day's session, a varnish-coated tungsten microelectrode with an initial impedance of several megaohms at $1 \mathrm{kHz}$ (Frederick Haer) was advanced vertically through the dura into the underlying cortex using a hydraulic microdrive (Narishige). The electrode could be placed reproducibly at points forming a square grid with $1 \mathrm{~mm}$ spacing. In the case of monkey $\mathrm{O}$, the action potentials of single neurons were isolated from the multineuronal trace by means of an on-line spikesorting system using a template matching algorithm (Signal Processing Systems). This spike-sorting system, on detection of an action potential, generated a pulse the time of which was stored with $1 \mathrm{~ms}$ resolution. In the case of monkey $\mathrm{T}$, three microelectrodes were inserted simultaneously at different grid locations, and single neurons from each microelectrode were sorted using on-line and off-line template matching and principal components analysis (Plexon).

\section{Recording sites}

All recording sites were within a region identified as the SEF on the basis of multiple criteria (Russo and Bruce, 1993, 2000). These criteria were as follows: (1) the site was located $4-8 \mathrm{~mm}$ rostral to the genu of the arcuate sulcus and 2-6 $\mathrm{mm}$ lateral to the midline as determined by structural MRI; (2) at the site or in its immediate vicinity, electrical microstimulation at a current strength $<80 \mu \mathrm{A}(100 \mathrm{~ms}$ train of biphasic pulses of 0.2 ms duration at $333 \mathrm{~Hz}$ ) elicited fixed vector or convergent eye movements; (3) neither at the site nor in its immediate vicinity did electrical microstimulation at current levels up to $200 \mu \mathrm{A}$ elicit orofacial or other bodily movements; (4) the site was located $\sim 1-2 \mathrm{~mm}$ rostral to the SMA and $\sim 1 \mathrm{~mm}$ lateral to the pre-SMA as identified on the basis of skeletomotor responses to electrical stimulation (Picard and Strick, 1996).

\section{Behavioral control and data collection}

All aspects of behavioral procedure, including presentation of stimuli, monitoring of eye movements, and delivery of reward, were under the control of a computer running Cortex software (National Institute of Mental Health; Cortex) in a DOS operating system. Eye position was monitored by means of a scleral search coil system (Riverbend Instruments). The $x$ and $y$ coordinates of eye position were stored at $4 \mathrm{~ms}$ intervals. Reward was delivered through a spigot under control of a solenoid valve on successful completion of each trial.

\section{Task design}

\section{Serial action task}

Basic design principle. On any given trial, the same image was placed at all three target locations. The identity of this image indicated in what order saccades must be made to the three locations.

Sequence of events in a trial. The monkey initiated a trial by acquiring central fixation. Two hundred and fifty milliseconds after attainment of fixation, three identical pictures $\left(\sim 4.5^{\circ}\right.$ across $)$ appeared at locations $11.4^{\circ}$ eccentric spaced at equal intervals of $120^{\circ}$ around fixation: straight up, down and to the right, and down and to the left. Disappearance of the central fixation spot after a variable interval (450-600 ms) signaled the monkey to make a saccade to the first location. One hundred milliseconds after completion of the saccade, a feedback stimulus appeared: a white annulus centered on the target. After a variable additional interval of eccentric fixation (225-350 ms), all peripheral stimuli vanished, and the central fixation spot reappeared, signaling the monkey to execute a saccade back to the center. After $150 \mathrm{~ms}$ of central fixation, the array of pictures reappeared, and the series of events was repeated with the sole exception that the saccade must be to second location. The third phase of the trial consisted of an equivalent series of events with the third location as the target. The trial terminated with $25 \mathrm{~ms}$ of central fixation followed by offset of all stimuli and delivery of reward. In the case of a saccade toward an incorrect location or a fixation break, the trial was aborted.

Number of conditions. Six different pictures instructed six different movement sequences. These represented all possible sequences in which the three locations could be, once each, successively selected.

Blocking. To reduce task difficulty, the monkey was allowed to complete a block of four successful trials under a given condition before another condition was imposed. Within a group of six successive blocks, each condition was represented once.

Number of trials. The session terminated when the monkey had completed 24 trials successfully under each of the six conditions.

Visually guided trials. In the event that the monkey selected an incorrect target on three successive trials, visual guidance was provided on the fourth trial by making the image at the location of the current target brighter than the other two. The frequency of trials on which visual guidance was provided, expressed as a percentage of all correct trials, was small (Monkey O: mean, 2.0\%; SD, 2.2\%; monkey T: mean, $15.4 \%$; SD, $6.2 \%)$. Visually guided trials were excluded from neuronal data analysis.

\section{Serial object task}

Basic design principle. The monkey was required to make saccades to three images (red cross, green hexagon, and blue triangle) in the same order on every trial. After each saccade, the images were subject to rearrangement. Thus, the monkey could not plan a fixed series of movements in advance.

Sequence of events in a trial. The sequence and timing of events and the spatial arrangement of stimuli were the same as in the serial action task.

Number of conditions. The current target (red cross during phase 1, green hexagon during phase 2, and blue triangle during phase 3 ) could occupy any of three locations. There were 27 conditions representing all possible sequences of target locations. The two nontarget images were assigned randomly to the two nontarget locations at each phase of the trial.

Number of trials. A session terminated when the monkey had completed four trials successfully under each of the 27 conditions.

\section{Variable-reward task}

Basic design principle. In the variable-reward task, the color and shape of the target informed the monkey whether the reward delivered on completion of the trial would be small (one drop of juice) or large (three drops).

Sequence of events in a trial. The monkey initiated a trial by acquiring central fixation. Shortly after attainment of fixation $(250 \mathrm{~ms})$, a cue indicating the size of reward (big or small) appeared at one of three fixed peripheral locations identical to the locations at which targets were placed in the serial order tasks. Disappearance of the central fixation spot (450-600 ms after the cue appeared) signaled the monkey to initiate a saccade toward the cue. One hundred milliseconds after completion of the saccade, a feedback stimulus appeared. This was a white annulus centered on the target. After an additional $225-350 \mathrm{~ms}$, all stimuli were turned off, and the monkey received a reward of the predicted size. In the event of a fixation break or in the absence of a saccade to the target, the trial was aborted.

Number of conditions. To dissociate neuronal selectivity for the visual attributes of the cue from selectivity for the size of the predicted reward, two different cues were used to signal a reward of each size. Crossing four cues with three locations gave 12 conditions.

Number of trials. A session terminated when the monkey had completed 10 trials successfully under each of the 12 conditions.

\section{Long-delay task}

Basic design principle. In the long-delay task, the interval between onset of the target and permission to execute a saccade had a duration (4.7 s) approximately equal to the duration of an entire trial in the serial action or serial object task.

Sequence of events in a trial. The sequence of events was the same as in the variable-reward task with the exception that the delay period had a duration of $4.7 \mathrm{~s}$. The target was a gray disk.

Number of conditions. There were three conditions in which the target appeared at the three possible locations.

Number of trials. A session terminated when the monkey had completed 20 trials successfully under each of the three conditions. 
Sequence of tasks

During recording from each site, the monkey first performed the serial action task and serial object task (in an order that alternated from site to site) and then performed the long-delay task followed by the variablereward task.

\section{Neuronal database}

We discontinued recording at a given site, if it appeared that neuronal activity was unmodulated during performance of the first task (always one of the serial order tasks) as indicated by inspection of raster and histogram displays. We restricted consideration to neurons characterized in the context of all four tasks. We further restricted consideration to neurons with activity that was task-related, when pooled across all trials, according to the following generous criterion: during at least one withintrial epoch in one task the firing rate should be significantly different $(t$ test; $p<0.05$ ) from the firing rate during a pretrial baseline period of 200 ms terminating with onset of the fixation spot. The within-trial epochs considered in this analysis were as follows.

\section{Serial order tasks}

(1) Three periarray epochs ( $100 \mathrm{~ms}$ before to $450 \mathrm{~ms}$ after onset of the array); (2) six perisaccade epochs (200 ms before to $300 \mathrm{~ms}$ after initiation of each centrifugal or centripetal saccade).

Variable-reward task

(1) Pericue epoch (100 ms before to $450 \mathrm{~ms}$ after onset of the cue; (2) perisaccade epoch (200 ms before to $300 \mathrm{~ms}$ after initiation of the saccade).

\section{Long-delay task}

Three equal epochs representing the beginning, middle, and final thirds of the full $4.7 \mathrm{~s}$ delay period.

Among 390 SEF neurons recorded in all four tasks (309 from monkey $\mathrm{T}$ and 81 from monkey O), 357 neurons ( 285 from monkey $\mathrm{T}$ and 72 from monkey O) were modulated in all four tasks. It was on these neurons that all subsequent data analysis was based.

\section{Counts of significant neuronal effects}

To characterize neuronal selectivity in the serial order tasks, we performed ANOVAs with firing rate as the dependent variable and with trial phase (first, second, or third) and direction (left, up, or right) as factors $(\alpha=0.05)$. Statements made in the main text are based specifically on the analysis of perisaccadic activity ( $200 \mathrm{~ms}$ before to $300 \mathrm{~ms}$ after initiation of the centrifugal saccade). The analysis of neuronal activity outside this epoch led to the same general conclusions (see supplemental material, Neuronal effects, available at www.jneurosci.org). To characterize neuronal selectivity in the variable-reward task, we performed an ANOVA with firing rate during the perisaccadic epoch (200 ms before to $300 \mathrm{~ms}$ after initiation of the saccade) as the dependent variable and with reward size (small or large) and direction (left, up, or right) as factors $(\alpha=0.05)$. To characterize neuronal selectivity in the long-delay task, we performed an ANOVA with firing rate as the dependent variable and with time (beginning, middle, or final third of the $4.7 \mathrm{~s}$ delay period) and direction (left, up, or right) as factors $(\alpha=0.05)$.

\section{Population histograms}

\section{Serial action task}

We parsed the data from each trial into six blocks. These were obtained by crossing three trial phases (first, second, and third) with two periods (array and saccade). The array period extended from $150 \mathrm{~ms}$ before to $450 \mathrm{~ms}$ after array onset. The saccade period extended from offset of the fixation spot to a point in time $600 \mathrm{~ms}$ later. This period encompassed the planning and execution of both the centrifugal and the centripetal saccades. Cutting out these blocks compensated for variability in trial duration arising from jitter introduced deliberately into the delay periods and from spontaneous variation in the behavioral reaction times. Having computed the average firing rate of each neuron in each $10 \mathrm{~ms}$ bin, we then computed the average across neurons of the firing rate in that bin.
For purposes of display, we spliced the six blocks into a continuous histogram. This was smoothed with a $20 \mathrm{~ms}$ Gaussian kernel.

\section{Serial object task}

The procedure was identical to the one described above for the serial action task.

\section{Variable-reward task}

We parsed the data from each trial into two blocks. The cue period extended from $150 \mathrm{~ms}$ before to $450 \mathrm{~ms}$ after target onset. The saccade period extended from offset of the fixation spot to a point in time $300 \mathrm{~ms}$ later. Having computed the average firing rate of each neuron in each 10 $\mathrm{ms}$ bin, we then computed the average across neurons of the firing rate in that bin. For purposes of display, we spliced the two blocks into a continuous histogram. This was smoothed with a $20 \mathrm{~ms}$ Gaussian kernel.

\section{Long-delay task}

Throughout the $4.7 \mathrm{~s}$ delay period after target onset, we computed the average firing rate of each neuron in each $10 \mathrm{~ms}$ bin. Then we computed the average across neurons of the firing rate in that bin. The resulting perievent histogram was smoothed with a $20 \mathrm{~ms}$ Gaussian kernel.

\section{Cross-task correlations}

\section{Serial object rank index versus serial action rank index}

On the basis of data from the serial action task, we computed, for each neuron, the mean firing rates during the perisaccade epochs $(200 \mathrm{~ms}$ before to $300 \mathrm{~ms}$ after initiation of the centrifugal saccade) of phase 1, phase 2, and phase 3: $A_{1}, A_{2}$, and $A_{3}$. We then normalized the values to their sum, so as to obtain a three-component normalized rank vector: $\left(A_{1}, A_{2}, A_{3}\right)$. By an identical procedure performed on firing rates from the serial object task, $\mathrm{O}_{1}, \mathrm{O}_{2}$, and $\mathrm{O}_{3}$, we obtained a second three-component normalized rank vector: $\left(\mathrm{O}_{1}, \mathrm{O}_{2}, \mathrm{O}_{3}\right)$. We computed the coefficient of correlation across neurons between the two rank vectors using the Matlab corrcoef function.

\section{Reward index versus serial action rank index}

On the basis of data from the serial action task, we computed for each neuron a normalized rank index. This was $\left(A_{3}-A_{1}\right) /\left(A_{3}+A_{1}\right)$, where $A_{1}$ and $A_{3}$ were the firing rates during the perisaccade epochs $(200 \mathrm{~ms}$ before to $300 \mathrm{~ms}$ after initiation of the centrifugal saccade) of phase 1 and phase 3. On the basis of data from the variable-reward task, we computed for each neuron a normalized reward index. This was $\left(\mathrm{R}_{\mathrm{b}}-\mathrm{R}_{\mathrm{s}}\right) /\left(\mathrm{R}_{\mathrm{b}}+\right.$ $R_{s}$ ), where $R_{s}$ and $R_{b}$ were the firing rates during the perisaccade epoch ( $200 \mathrm{~ms}$ before to $300 \mathrm{~ms}$ after initiation of the saccade) on small-reward and big-reward trials. We then computed the coefficient of correlation across neurons between the rank and reward vectors using the Matlab corrcoef function.

\section{Time index versus serial action rank index}

On the basis of data from the serial action task, we computed, for each neuron, the mean firing rates during the perisaccade epochs (200 ms before to $300 \mathrm{~ms}$ after initiation of the centrifugal saccade) of phase 1 , phase 2, and phase 3: $A_{1}, A_{2}$, and $A_{3}$. We then normalized the values to their sum, so as to obtain a three-component normalized rank vector: $\left(A_{1}, A_{2}, A_{3}\right)$. By an identical procedure performed on firing rates from the beginning, middle, and final thirds of the $4.7 \mathrm{~s}$ delay period of the longdelay task, $\mathrm{T}_{1}, \mathrm{~T}_{2}$, and $\mathrm{T}_{3}$, we obtained a second three-component normalized rank vector: $\left(T_{1}, T_{2}, T_{3}\right)$. We computed the coefficient of correlation across neurons between the two rank vectors using the Matlab corrcoef function.

\section{Partial correlation analysis}

For each neuron, we computed four indices: a rank index in the serial action task: $\left(\mathrm{A}_{3}-\mathrm{A}_{1}\right) /\left(\mathrm{A}_{3}+\mathrm{A}_{1}\right)$; a rank index in the serial object task $\left(\mathrm{O}_{3}\right.$ $\left.-\mathrm{O}_{1}\right) /\left(\mathrm{O}_{3}+\mathrm{O}_{1}\right)$; a reward index in the variable-reward task $\left(\mathrm{R}_{\mathrm{b}}-\right.$ $\left.\mathrm{R}_{\mathrm{s}}\right) /\left(\mathrm{R}_{\mathrm{b}}+\mathrm{R}_{\mathrm{s}}\right)$; and a time index in the long-delay task $\left(\mathrm{T}_{3}-\mathrm{T}_{1}\right) /\left(\mathrm{T}_{3}+\right.$ $\mathrm{T}_{1}$ ). These are defined in terms of variables defined above. We then computed the partial correlation between the rank indices in the serial order tasks (with the reward index and the time index as control variables) using the Matlab partialcorr function. 


\section{A. Serial action task}
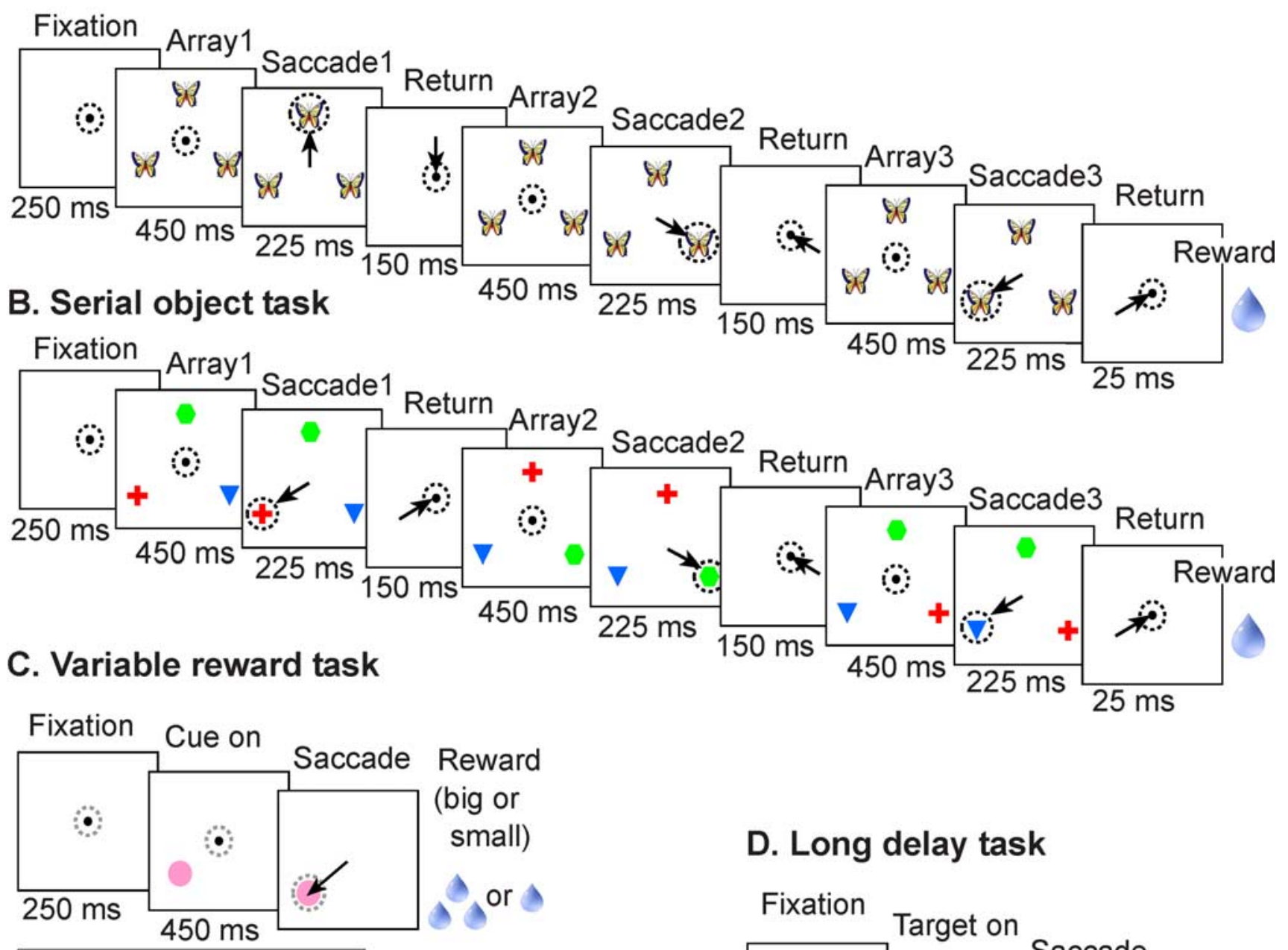

\section{Long delay task}

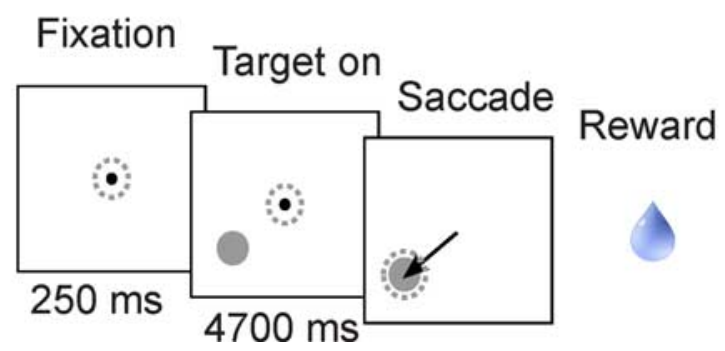

Figure 1. Sequence of events in a typical trial in each of the four tasks. Arrows indicate saccades and broken circles indicate direction of gaze. The time indicated beneath each panel is the minimal possible duration of fixation during the corresponding stage of the trial. $A$, Serial action task. After $250 \mathrm{~ms}$ of acquiring central fixation, three identical pictures appeared at three fixed locations. The identity of the picture served as a sequence cue. Disappearance of the central fixation spot after a variable interval $(450-600 \mathrm{~ms})$ signaled the monkey to make a saccade to the first location. The saccadic reaction time varied around a mean of $\sim 150 \mathrm{~ms}$. One hundred milliseconds after completion of the saccade, a feedback stimulus appeared (data not shown); after a variable additional interval of eccentric fixation $(225-350 \mathrm{~ms})$, all peripheral stimuli vanished, and the central fixation spot reappeared, signaling the monkey to execute a saccade back to the center. The saccadic reaction time varied around a mean of $\sim 150 \mathrm{~ms}$. After $150 \mathrm{~ms}$ of central fixation, the array of pictures reappeared, and the series of events was repeated with the sole exception that the saccade must be to the second location. The third phase of the trial consisted of an equivalent series of events with the third location as the target. The trial terminated with $25 \mathrm{~ms}$ of central fixation followed by offset of all stimuli and delivery of reward. In the case of a saccade toward an incorrect location or a fixation break, the trial was aborted. For further details, see Materials and Methods, Task design. $B$, Serial object task. The monkey was required to make saccades to three images (red cross, green hexagon, and blue triangle) in the same order on every trial. After each saccade, the images were subject to rearrangement. The sequence and timing of events and the spatial arrangement of stimuli were the same as in the serial action task. C, Variable-reward task. The color and shape of the target informed the monkey whether the reward delivered on completion of the trial would be small (one drop of juice) or large (three drops). The monkey initiated a trial by acquiring central fixation. Shortly after attainment of fixation $(250 \mathrm{~ms})$, a cue indicating the size of reward (big or small) appeared at one of three fixed peripheral locations identical to the locations at which targets were placed in the serial order tasks. Disappearance of the central fixation spot ( $450-600 \mathrm{~ms}$ after the cue appeared) signaled the monkey to initiate a saccade toward the cue. One hundred milliseconds after completion of the saccade, a feedback stimulus appeared. This was a white annulus centered on the target. After an additional $225-350 \mathrm{~ms}$, all stimuli were turned off, and the monkey received a reward of the predicted size. In the event of a fixation break or in the absence of a saccade to the target, the trial was aborted. $\boldsymbol{D}$, Long-delay task. The sequence of events was the same as in the variable-reward task with the exception that the delay period had a duration of $4.7 \mathrm{~s}$, approximately equal to the duration of an entire trial in the serial action or serial object task. The target was a gray disk.

\section{Results}

We studied the activity of 357 SEF neurons (285 from monkey T and 72 from monkey $\mathrm{O}$ ) during performance of both a serial action task and a serial object task. The serial action task (Fig. 1A) required making saccades to three targets at standard locations. The six possible sequences were signaled by six images. For example, if images of a butterfly occupied the three target locations, then the monkey had to make first an upward, then a rightward, 
Table 1. Neuronal effects in the serial action task (ANOVA; $\alpha=0.05$ )

\begin{tabular}{lccccc}
\hline Serial action task & Recorded & Selected & Rank & Direction & $\begin{array}{l}\text { Rank } \times \text { direction } \\
\text { interaction }\end{array}$ \\
\hline Monkey 0 & 81 & 72 & 59 & 41 & 30 \\
Monkey T & 309 & 285 & 262 & 233 & 205 \\
Total & 390 & 357 & 321 & 274 & 235 \\
\% of total selected & & 100 & 90 & 77 & 66 \\
\hline
\end{tabular}

and finally a leftward saccade. Completion of the sequence was rewarded with a drop of juice. Both monkeys performed at a high level, with an average percentage correct score $>94 \%$ (see supplemental material, Behavioral performance, available at www.jneurosci.org).

To characterize the dependence of each neuron's firing rate on rank and saccade direction, we performed a two-factor ANOVA $(\alpha=0.05)$ with saccade direction and rank as factors and with firing rate as the dependent variable. Although the main focus of this study is on rank selectivity, we also characterized neuronal selectivity for saccade direction, because it forms a useful baseline against which to compare selectivity for rank. In agreement with previous studies (Russo and Bruce, 2000; Isoda and Tanji, 2002, 2003; Lu et al., 2002; Roesch and Olson, 2003; Uchida et al., 2007), we found that the activity of a majority of SEF neurons (274 of $357 ; 77 \%$ ) varied as a main effect of saccade direction (Table 1). However, we found that even more neurons (321 of $357 ; 90 \%$ ) exhibited a main effect of rank and that many neurons (235 of 357; 66\%) exhibited a direction-rank interaction effect (Table 1). It is important to note that neuronal selectivity for rank could not be attributed to the directional sensitivity, because the task incorporated full counterbalancing between saccade direction and rank. For each rank-selective neuron, we identified the preferred rank as the stage of the trial during which it fired most strongly. Among 321 neurons, 51 preferred the first, 115 the second, and 155 the third rank. An example of a neuron preferring the second rank is shown in Figure $2 A$. The timing and strength of activity among neurons preferring each rank can be judged from the population histograms of Figure $3 \mathrm{~A}$.

To determine whether rank selectivity persisted outside the context of the serial action task, we recorded the activity of the same 357 neurons in the context of the serial object task (Figure $1 B$ ). On each trial of this task, the monkey had to make a saccade first to the red cross, then to the green hexagon, and finally to the blue triangle. The arrangement of the three targets was scrambled from phase to phase of the trial so that it was impossible for the monkey to complete the trial successfully by planning and carrying out a sequence of actions. Both monkeys performed at a high level, with an average percentage correct score $>86 \%$ (see supplemental material, Behavioral performance, available at www.jneurosci.org).

In this task, as in the previous one, many SEF neurons (262 of $357 ; 74 \%$ ) were selective for saccade direction (ANOVA; main effect of direction; $p<0.05$ ). However, even more neurons (301 of 357; 84\%) were rank-selective (ANOVA; main effect of rank; $p<0.05)$ and many exhibited a direction-rank interaction (203 of 357, 57\%; ANOVA; $p<0.05$ ) (Table 2). If a given neuron was rank-selective in the serial action task, then it tended to be rankselective in the serial object task. This trend was significantly greater than expected by chance $\left(\chi^{2}\right.$ test; $\left.p<0.0001\right)$. Finally, if a neuron preferred a given rank in the serial action task then it tended to prefer the same rank in the serial object task. This principle is evident both in the example of Figure 2, $A$ and $B$, and in the population histograms of Figure $3 B$, where neurons se-

\section{A. Serial action task}

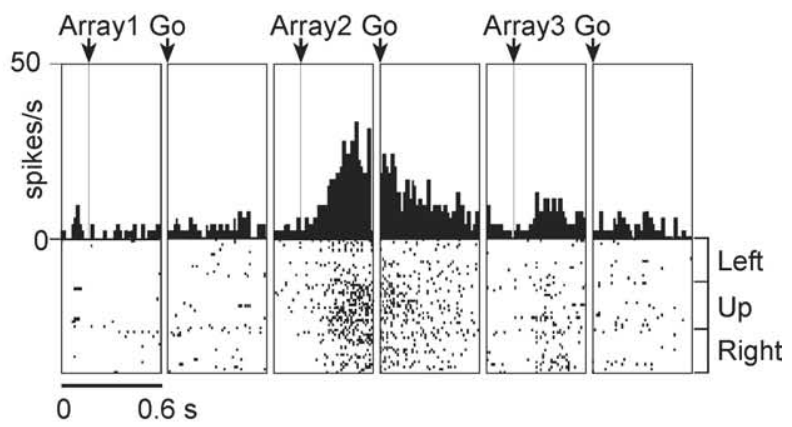

B. Serial object task

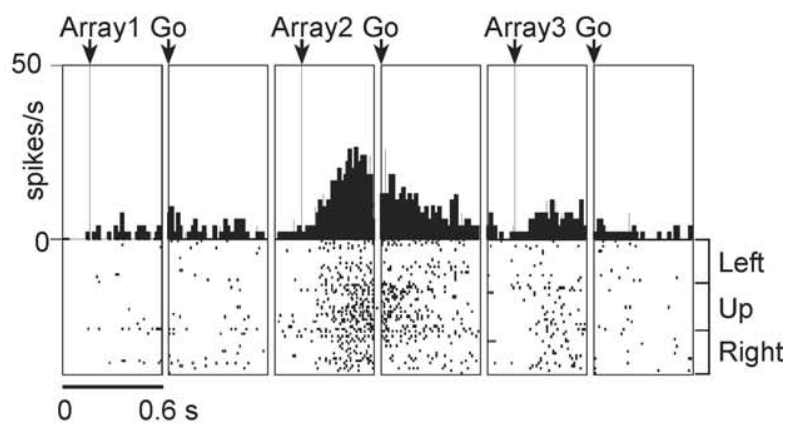

C. Variable reward task Small reward
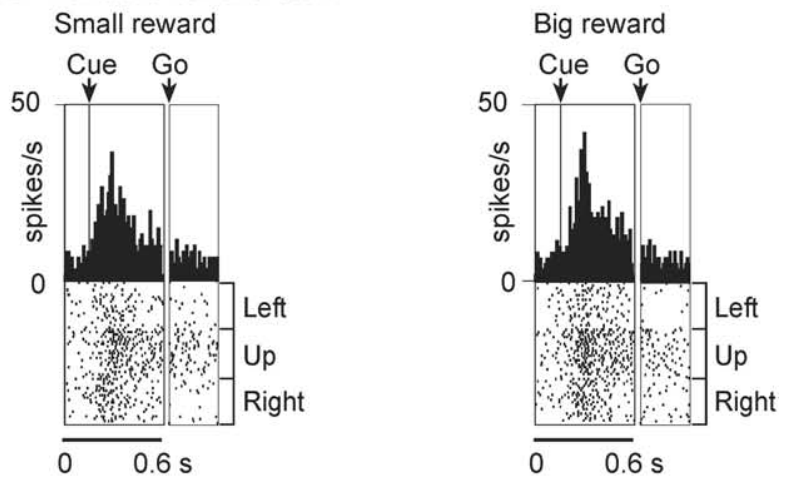

D. Long delay task

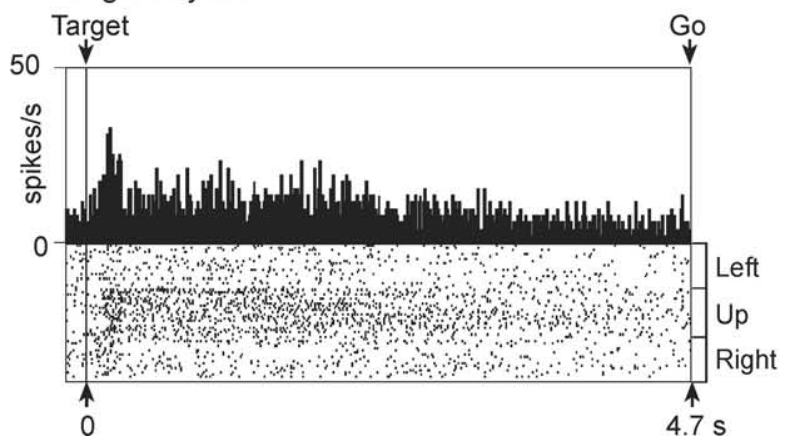

Figure 2. Neuron with concordant rank selectivity in two serial order tasks. $A$, Serial action task. Maximal firing during the second trial phase. Data in the "Array" panels are aligned on array presentation. Data in the "Go" panels are aligned on the signal for the centrifugal saccade (offset of the central fixation spot). $B$, Serial object task. Maximal firing during the second trial phase. $C$, Variable-reward task. Firing rate is mostly unaffected by expected reward size. Data are aligned on the cue presentation in "Cue" panels and fixation spot offset in "Go" panels. $\boldsymbol{D}$, Long-delay task. A weak trend toward maximal firing in the beginning of the trial. Data are aligned on target onset. If selectivity for rank 2 in the serial order tasks $(\boldsymbol{A}, \boldsymbol{B})$ had depended on neuronal sensitivity to the passage of time, this neuron would have fired maximally in the middle of the long delay period. 
lected for exhibiting first, second, or third rank selectivity on the basis of their activity in the serial action task exhibit concordant selectivity in the serial object task. To assess the statistical significance of the trend toward concordance, we first tested whether the proportion of rank-selective neurons preferring the same rank in both tasks was significantly higher than expected on the assumption of independent distributions ( $\chi^{2}$ test; $\alpha=0.05$ ). We found that among neurons exhibiting rank selectivity in both tasks ( 280 of $357 ; 78 \%$ of all studied neurons), cases of cross-task agreement in preferred rank were significantly more numerous than expected on the assumption of independence ( $\chi^{2}$ test; $\left.p<10^{-6}\right)$. To supplement the countbased test, we used an analysis based on a continuous measure (see Materials and Methods, Cross-task correlations). For each neuron, we computed the mean firing rate during each trial phase of the serial action task by averaging firing rates across different directions. We then normalized the values to their sum, so as to obtain a three-component normalized rank vector: $\left(A_{1}, A_{2}, A_{3}\right)$. By an identical procedure performed on firing rates from the serial object task, we obtained a second threecomponent normalized rank vector: $\left(O_{1}\right.$, $\left.\mathrm{O}_{2}, \mathrm{O}_{3}\right)$. By pooling the pairs of rank vectors across all 357 neurons, we analyzed the correlation across neurons between the rank preferences in the serial action task and rank preferences in the serial object task. The correlation was positive and highly significant (Fig. 4A) $\left(R^{2}=0.55 ; p<0.0001\right)$.

Although it is certain that the firing of SEF neurons was consistently correlated with rank across the two tasks, one might still question whether it was rank per se to which the neurons were sensitive or some other factor correlated with rank. One such factor was reward expectation. This presumably increased steadily as the trial progressed from the first through the second to the third phase. To assess the impact of reward expectation, we recorded from neurons during performance of the variablereward task (Fig. 1C). In this task, a cue presented early in the trial indicated whether three drops or one drop of juice would be delivered after the saccade at the trial's end. In 177 of 357 neurons $(50 \%)$, the firing rate was significantly affected by reward expectation (ANOVA; main effect of reward size; $p<0.05$ ). Among these neurons, 86 fired more strongly under the small-reward condition and 91 under the large-reward condition. On the assumption that rank-related firing in the serial order tasks arose from neuronal sensitivity to the intensity of reward expectation (presumably least during trial phase 1 and greatest during trial phase 3 ), we would expect that neurons firing most during phase 1 or phase 3 in the serial order tasks should fire most in expectation of a small or a large reward in the variable-reward task. However, we found that there was only a weak linkage between the two phenomena. Rank-selective neurons were not significantly more likely than other neurons to exhibit selectivity for reward size ( $\chi^{2}$ test; $p=0.26$ ). At the level of population activity,

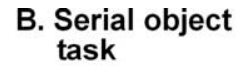

C. Variable

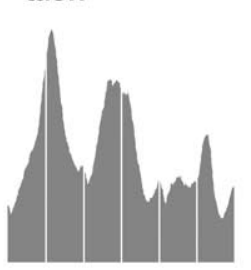

reward task
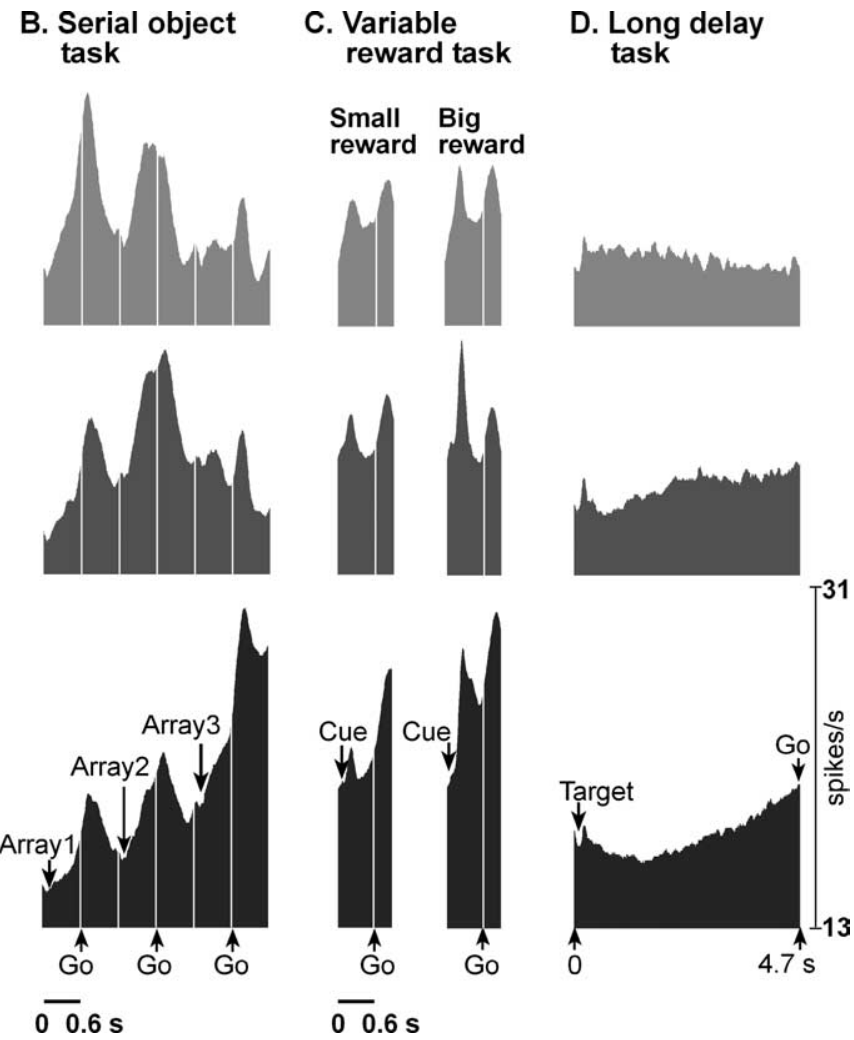

$00.6 \mathrm{~s}$

Figure 3. Population activity of rank selective neurons. Mean firing rate as a function of time for SEF neurons classified, on the basis of activity in the serial action task, as selective for the first, second, or third rank. For conventions governing alignment of the data, see legend to Figure 2. A, Serial action task. Neurons selective for first, second, and third rank by definition exhibit maxima serial action task $(\boldsymbol{A})$ exhibit concordant selectivity in the serial object task. $\boldsymbol{C}$, Variable-reward task. In the population selective for 3, there is a slight tendency for firing to be stronger in anticipation of a big reward. $\boldsymbol{D}$, Long-delay task. In populations selective for ranks 1 and 3, there is a slight tendency for firing to decline and increase over the course of the delay period.

Table 2. Neuronal effects in the serial object task (ANOVA; $\alpha=0.05$ )

\begin{tabular}{lccccc}
\hline Serial object task & Recorded & Selected & Rank & Direction & $\begin{array}{l}\text { Rank } \times \text { direction } \\
\text { interaction }\end{array}$ \\
\hline Monkey 0 & 81 & 72 & 57 & 49 & 42 \\
Monkey T & 309 & 285 & 244 & 216 & 161 \\
Total & 390 & 357 & 301 & 262 & 203 \\
\% of total selected & & 100 & 84 & 74 & 57 \\
\hline
\end{tabular}

there was a tendency for neurons favoring the third rank to fire more strongly in anticipation of a large reward but no tendency for neurons favoring the first rank to fire more strongly in anticipation of a small reward (Fig. $3 A-C$ ). Finally, the correlation across neurons between the rank index (serial action task: firing during phase 3 minus firing during phase 1 normalized to the sum) and the reward index (variable-reward task: firing in anticipation of large reward minus firing in anticipation of small reward normalized to the sum), although significant, was small (Fig. $4 B)\left(R^{2}=0.13 ; p<0.0001\right)$.

Another factor correlated with rank is the passage of time. Necessarily, the first, second, and third phases of a serial order trial coincide with its beginning, middle, and end. To assess the impact of this factor, we recorded neuronal activity during performance of a long-delay task (Fig. $1 D$ ) requiring the monkey to maintain central fixation during an interval (4700 ms) matched in duration to an entire trial in the serial action or serial object task. For purposes of analysis, we divided the delay period into beginning, middle, and end segments corresponding to the first, 

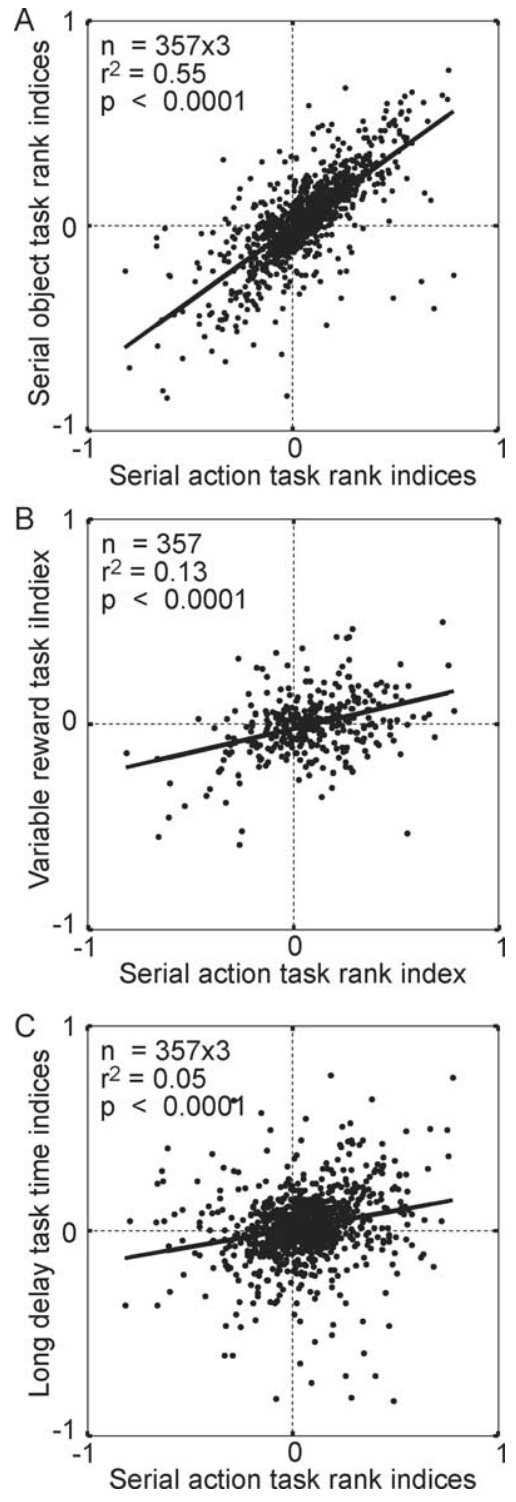

Figure 4. Correlation between indices of selectivity measured in different tasks. $\boldsymbol{A}$, Rank index in serial object task versus rank index in serial action task. Correlation analysis was performed on three-component vectors as described in Materials and Methods, Cross-task correlations. However, for graphic presentation, each neuron contributed three indices from each task: $(2-1) /(2+1),(3-1) /(3+1)$ and $(3-2) /(3+2)$, where 1,2 , and 3 represent firing rates during trial phases 1,2 , and 3 , respectively. $B$, Reward index in variable-reward task versus rank index in serial action task. Reward index: $(B-S) /(B+S)$, where $B$ and $S$ represent the firing rates during big-reward and small-reward trials. Rank index: $(3-1) /(3+1)$, where 1 and 3 are defined as under $\boldsymbol{A}$ above. $\boldsymbol{C}$, Time index in long-delay task versus rank index in serial action task. Correlation analysis was performed on three-component vectors as described in Materials and Methods, Cross-task correlations. However, for graphic presentation, each neuron contributed three indices from the long-delay task $(M-B) /(M+B),(E-B) /(E+B)$, and $(E-M) /(E+M)$, where $B, M$, and $E$ represent firing rates during the beginning, middle, and end of the delay period, and three corresponding rank indices from the serial action task $(2-1) /(2+$ $1),(3-1) /(3+1)$, and $(3-2) /(3+2)$, where 1,2 , and 3 are defined as under $A$ above.

second, and third phases of the serial action task. We then assessed whether the dependence of firing rate on time in this task was related to its dependence on rank in the serial action task. A total of 254 of 357 neurons (71\%) exhibited a significant effect of time (ANOVA; main effect of period; $p<0.050$ ). Across the neuronal population as a whole, the linkage between rank selectivity and time selectivity was weak. The tendency for neurons with rank selectivity to exhibit time selectivity did not achieve significance $\left(\chi^{2}\right.$ test; $p=0.11$ ). Plots of population activity in the long-delay task showed a definite tendency for firing to ramp up over time in neurons selective for the third rank but only a weak tendency for firing to decline over time in neurons selective for the first rank (Fig. 3D). Finally, although there was a significant positive correlation across neurons between the threecomponent vector representing time-selectivity and the threecomponent vector representing rank selectivity, the correlation coefficient was very small (Fig. $4 C)\left(R^{2}=0.05 ; p<0.0001\right)$.

As a final check on whether the correlation between rank selectivity in the serial action task and the serial object task could be accounted for by factors other than rank, we factored out, in a partial correlation analysis, the variance explained by reward size and time. The correlation remained strong, positive, and highly significant (partial $R^{2}=0.498$ compared with initial $R^{2}=0.561$ ). We conclude that SEF neurons are rank generalists, consistently representing rank (as distinct from the approach of reward or the passage of time) across tasks that require the execution of a learned series of movements and the selection of objects in a learned sequence.

\section{Discussion}

The first key conclusion of this study is that SEF neurons exhibit rank selectivity during the performance of a task requiring the monkey to select a series of objects in a learned sequence without regard to their location. It has been noted previously that SEF neurons exhibit rank selectivity in the tasks requiring execution of a memorized sequence of eye movements (Isoda and Tanji, 2002 , 2003). However, before the current study, it was not known whether neurons in the SEF or anywhere else in the brain exhibit ordinal position selectivity in conjunction with selecting objects in a memorized sequence. In the few previous neurophysiological studies using a serial object task (Ninokura et al., 2003, 2004; Inoue and Mikami, 2006), prefrontal neurons were shown to exhibit rank selectivity during presentation of a series of sample objects under conditions of steady fixation. However, neuronal activity accompanying subsequent selection of the objects was not described.

The second key conclusion is that SEF neurons exhibit concordant ordinal position selectivity in the serial action task and the serial object task. This means (1) that rank selectivity in the SEF does not depend on the monkey's performing a learned series of movements and (2) that SEF neurons, far from being segregated into populations responsible for representing rank in different behavioral contexts, generalize across task contexts. No previous study has pointed toward this conclusion because none has characterized neuronal activity in the context of two serial order tasks.

Our third conclusion is that rank selectivity is not an artifact of neuronal sensitivity to the growing expectation of reward. This seemed possible because, in the standard serial order task design, the monkey expects to receive reward after completion of the sequence (Kermadi and Joseph, 1995; Funahashi et al., 1997; Clower and Alexander, 1998; Carpenter et al., 1999; Shima and Tanji, 2000; Procyk and Joseph, 2001; Averbeck et al., 2002, 2003; Akkal et al., 2002; Isoda and Tanji, 2002, 2003; Lu et al., 2002; Lee and Quessy, 2003; Ohbayashi et al., 2003; Inoue and Mikami, 2006; Sohn and Lee, 2007). Furthermore, it has been a concern because the same brain areas that contain rank-selective neurons also contain neurons sensitive to the anticipation and delivery of reward (Amador et al., 2000; Roesch and Olson, 2003, 2004, 2005a,b, 2007; Wallis and Miller, 2003; Campos et al., 2005; Uchida et al., 2007; Ichihara-Takeda and Funahashi, 2008). By 
comparing, for the first time, the sensitivity of the same neurons to rank in a serial order task and to reward expectation in a task involving predictable rewards of variable size, we have been able to show that, at least in the SEF, the dependence of firing rate on rank cannot be explained by its dependence on the expectation of reward.

A final observation is that rank selectivity is not an artifact of neuronal sensitivity to the passage of time. This seemed possible because progress from phase to phase within the trial has been confounded with the passage of time in all studies of serial order performance performed to date (Kermadi and Joseph, 1995; Funahashi et al., 1997; Clower and Alexander, 1998; Carpenter et al., 1999; Shima and Tanji, 2000; Procyk and Joseph, 2001; Averbeck et al., 2002, 2003; Akkal et al., 2002; Isoda and Tanji, 2002, 2003; Lu et al., 2002; Lee and Quessy, 2003; Ohbayashi et al., 2003; Inoue and Mikami, 2006; Sohn and Lee, 2007). Furthermore, it has been a concern because the same brain areas that contain rank-selective neurons also contain neurons with activity that varies over the course of a delay period, often ramping up or down (Kojima and Goldman-Rakic, 1982; Quintana and Fuster, 1992; Miller et al., 1996; Watanabe, 1996; Heinen and Liu, 1997; Chafee and Goldman-Rakic, 1998; Kawagoe et al., 1998; Rainer et al., 1999; Hassani et al., 2001; Brody et al., 2003; Akkal et al., 2004; Reutimann et al., 2004; Roesch and Olson, 2005a,b; Tsujimoto and Sawaguchi, 2005; Genovesio et al., 2006). By comparing, for the first time, the sensitivity of the same neurons to rank in a serial order task and to the passage of time in a task with a long delay, we have been able to show that, at least in the SEF, the dependence of firing rate on rank cannot be explained by its dependence on the passage of time. It is possible that neuronal sensitivity to the passage of time would have been stronger if we had demarcated the segments of the delay period by requiring the monkey to make saccades (although not in accordance with a serial order rule) at instants when saccades were executed in the serial order task (Hasegawa et al., 2004). However, a given neuron's pattern of selectivity would not likely have been altered by this manipulation, and, thus, the degree of concordance between rank and time signals would not likely have been improved.

\section{References}

Akkal D, Bioulac B, Audin J, Burbaud P (2002) Comparison of neuronal activity in the rostral supplementary and cingulate motor areas during a task with cognitive and motor demands. Eur J Neurosci 15:887-904.

Akkal D, Escola L, Bioulac B, Burbaud P (2004) Time predictability modulates pre-supplementary motor area neuronal activity. Neuroreport 15:1283-1286.

Amador N, Schlag-Rey M, Schlag J (2000) Reward-predicting and rewarddetecting neuronal activity in the primate supplementary eye field. J Neurophysiol 84:2166-2170.

Ashe J, Lungu OV, Basford AT, Lu X (2006) Cortical control of motor sequences. Curr Opin Neurobiol 16:213-221.

Averbeck BB, Chafee MV, Crowe DA, Georgopoulos AP (2002) Parallel processing of serial movements in prefrontal cortex. Proc Natl Acad Sci U S A 99:13172-13177.

Averbeck BB, Crowe DA, Chafee MV, Georgopoulos AP (2003) Neural activity in prefrontal cortex during copying geometrical shapes. II. Decoding shape segments from neural ensembles. Exp Brain Res 150:142-153.

Brody CD, Hernández A, Zainos A, Romo R (2003) Timing and neural encoding of somatosensory parametric working memory in macaque prefrontal cortex. Cereb Cortex 13:1196-1207.

Campos M, Breznen B, Bernheim K, Andersen RA (2005) Supplementary motor area encodes reward expectancy in eye-movement tasks. J Neurophysiol 94:1325-1335.

Carpenter AF, Georgopoulos AP, Pellizzer G (1999) Motor cortical encoding of serial order in a context-recall task. Science 283:1752-1757.

Chafee MV, Goldman-Rakic PS (1998) Matching patterns of activity in pri- mate prefrontal area $8 \mathrm{a}$ and parietal area 7ip neurons during a spatial working memory task. J Neurophysiol 79:2919-2940.

Clower WT, Alexander GE (1998) Movement sequence-related activity reflecting numerical order of components in supplementary and presupplementary motor areas. J Neurophysiol 80:1562-1566.

Crowe DA, Chafee MV, Averbeck BB, Georgopoulos AP (2004) Participation of primary motor cortical neurons in a distributed network during maze solution: representation of spatial parameters and time-course comparison with parietal area 7a. Exp Brain Res 158:28-34.

Dominey PF, Hoen M, Blanc JM, Lelekov-Boissard T (2003) Neurological basis of language and sequential cognition: evidence from simulation, aphasia, and ERP studies. Brain Lang 86:207-225.

Funahashi S, Inoue M, Kubota K (1997) Delay-period activity in the primate prefrontal cortex encoding multiple spatial positions and their order of presentation. Behav Brain Res 84:203-223.

Genovesio A, Tsujimoto S, Wise SP (2006) Neuronal activity related to elapsed time in prefrontal cortex. J Neurophysiol 95:3281-3285.

Hasegawa RP, Blitz AM, Goldberg ME (2004) Neurons in monkey prefrontal cortex whose activity tracks the progress of a three-step self-ordered task. J Neurophysiol 92:1524-1535.

Hassani OK, Cromwell HC, Schultz W (2001) Influence of expectation of different rewards on behavior-related neuronal activity in the striatum. J Neurophysiol 85:2477-2489.

Heinen SJ, Liu M (1997) Single-neuron activity in the dorsomedial frontal cortex during smooth-pursuit eye movements to predictable target motion. Vis Neurosci 14:853-865.

Hoen M, Golembiowski M, Guyot E, Deprez V, Caplan D, Dominey PF (2003) Training with cognitive sequences improves syntactic comprehension in agrammatic aphasics. Neuroreport 14:495-499.

Ichihara-Takeda S, Funahashi S (2008) Activity of primate orbitofrontal and dorsolateral prefrontal neurons: effect of reward schedule on taskrelated activity. J Cogn Neurosci 20:563-579.

Inoue M, Mikami A (2006) Prefrontal activity during serial probe reproduction task: encoding, mnemonic, and retrieval processes. J Neurophysiol 95:1008-1041.

Isoda M, Tanji J (2002) Cellular activity in the supplementary eye field during sequential performance of multiple saccades. J Neurophysiol 88:3541-3545.

Isoda M, Tanji J (2003) Contrasting neuronal activity in the supplementary and frontal eye fields during temporal organization of multiple saccades. J Neurophysiol 90:3054-3065.

Kawagoe R, Takikawa Y, Hikosaka O (1998) Expectation of reward modulates cognitive signals in the basal ganglia. Nat Neurosci 1:411-416.

Kermadi I, Joseph JP (1995) Activity in the caudate nucleus of monkey during spatial sequencing. J Neurophysiol 74:911-933.

Kojima S, Goldman-Rakic PS (1982) Delay-related activity of prefrontal neurons in rhesus monkeys performing delayed response. Brain Res 248:43-49.

Lashley K (1951) The problem of serial order in behavior. In: Cerebral mechanisms in behavior (Jeffress L, ed), pp 112-147. New York: Wiley.

Lee D, Quessy S (2003) Activity in the supplementary motor area related to learning and performance during a sequential visuomotor task. J Neurophysiol 89:1039-1056.

Lu X, Ashe J (2005) Anticipatory activity in primary motor cortex codes memorized movement sequences. Neuron 45:967-973.

Lu X, Matsuzawa M, Hikosaka O (2002) A neural correlate of oculomotor sequences in supplementary eye field. Neuron 34:317-325.

Miller EK, Erickson CA, Desimone R (1996) Neural mechanisms of visual working memory in prefrontal cortex of the macaque. J Neurosci 16:5154-5167.

Mushiake H, Strick PL (1995) Pallidal neuron activity during sequential arm movements. J Neurophysiol 74:2754-2758.

Ninokura Y, Mushiake H, Tanji J (2003) Representation of the temporal order of visual objects in the primate lateral prefrontal cortex. J Neurophysiol 89:2868-2873.

Ninokura Y, Mushiake H, Tanji J (2004) Integration of temporal order and object information in the monkey lateral prefrontal cortex. J Neurophysiol 91:555-560.

Ohbayashi M, Ohki K, Miyashita Y (2003) Conversion of working memory to motor sequence in the monkey premotor cortex. Science 301:233-236.

Picard N, Strick PL (1996) Motor areas of the medial wall: a review of their location and functional activation. Cereb Cortex 6:342-353. 
Procyk E, Joseph JP (2001) Characterization of serial order encoding in the monkey anterior cingulate sulcus. Eur J Neurosci 14:1041-1046.

Quintana J, Fuster JM (1992) Mnemonic and predictive functions of cortical neurons in a memory task. Neuroreport 3:721-724.

Rainer G, Rao SC, Miller EK (1999) Prospective coding for objects in primate prefrontal cortex. J Neurosci 19:5493-5505.

Reutimann J, Yakovlev V, Fusi S, Senn W (2004) Climbing neuronal activity as an event-based cortical representation of time. J Neurosci 24:3295-3303.

Rhodes BJ, Bullock D, Verwey WB, Averbeck BB, Page MP (2004) Learning and production of movement sequences: behavioral, neurophysiological, and modeling perspectives. Hum Mov Sci 23:699-746.

Roesch MR, Olson CR (2003) Impact of expected reward on neuronal activity in prefrontal cortex, frontal and supplementary eye fields and premotor cortex. J Neurophysiol 90:1766-1789.

Roesch MR, Olson CR (2004) Neuronal activity related to reward value and motivation in primate frontal cortex. Science 304:307-310.

Roesch MR, Olson CR (2005a) Neuronal activity dependent on anticipated and elapsed delay in macaque prefrontal cortex, frontal and supplementary eye fields, and premotor cortex. J Neurophysiol 94:1469-1497.

Roesch MR, Olson CR (2005b) Neuronal activity in primate orbitofrontal cortex reflects the value of time. J Neurophysiol 94:2457-2471.

Roesch MR, Olson CR (2007) Neuronal activity related to anticipated reward in frontal cortex: does it represent value or reflect motivation? Ann N Y Acad Sci 1121:431-446.

Russo GS, Bruce CJ (1993) Effect of eye position within the orbit on electri- cally elicited saccadic eye movements: a comparison of the macaque monkey's frontal and supplementary eye fields. J Neurophysiol 69:800-818.

Russo GS, Bruce CJ (2000) Supplementary eye field: representation of saccades and relationship between neural response fields and elicited eye movements. J Neurophysiol 84:2605-2621.

Shima K, Tanji J (2000) Neuronal activity in the supplementary and presupplementary motor areas for temporal organization of multiple movements. J Neurophysiol 84:2148-2160.

Sohn JW, Lee D (2007) Order-dependent modulation of directional signals in the supplementary and presupplementary motor areas. J Neurosci 27:13655-13666.

Tanji J (2001) Sequential organization of multiple movements: involvement of cortical motor areas. Annu Rev Neurosci 24:631-651.

Tsujimoto S, Sawaguchi T (2005) Neuronal activity representing temporal prediction of reward in the primate prefrontal cortex. J Neurophysiol 93:3687-3692.

Uchida Y, Lu X, Ohmae S, Takahashi T, Kitazawa S (2007) Neuronal activity related to reward size and rewarded target position in primate supplementary eye field. J Neurosci 27:13750-13755.

Wallis JD, Miller EK (2003) Neuronal activity in primate dorsolateral and orbital prefrontal cortex during performance of a reward preference task. Eur J Neurosci 18:2069-2081.

Watanabe M (1996) Reward expectancy in primate prefrontal neurons. Nature 382:629-632. 